\title{
Mobile Specification Using Semantic Networks
}

\author{
Haeng-Kon Kim \\ Department of Computer Information \& Communication Engineering, \\ Catholic University of Daegu, Kyungbuk, 712-702, South Korea \\ hangkon@cu.ac.kr
}

\begin{abstract}
A semantic network is a network which represents semantic relations among concepts. This is often used as a form of knowledge representation.This paper introduces a retrieval method based on semantic networks. A hyperlink information is essential to construct semantic networks. The information is very useful as it provides summary and further linkage to construct Semantic networks that has been provided by human. It also has a property which shows review, relation, hierarchy, generality, and visibility. Using this property, we extracted the keywords of mobile documents and made up of the Semantic networks among the keywords sampled from mobile pages. This paper extracts the keywords of mobile pages using anchor text one out of hyperlink information and makes hyperlink of mobile pages abstract as the link relation between keywords of each mobile page. I suggest this useful retrieval method providing querying word extension or domain knowledge by Semantic networks of keywords.
\end{abstract}

\section{References}

1. Berry, M.J.A., Linoff, G.: Link analysis. In: Data Mining Techniques: For marketing, Sales, and Customer Support, pp. 216-242. Wiley Computer Publishing, Chichester (1998)

2. Brin, S., Page, L.: The Anatomy of a Large-Scale Hyper textual Mobile Search Engine. In: Proceeding of the Seventh International World Wide Mobile Conference (2002)

3. Callan, J.P., Croft, W.B., Harding, S.M.: The INQUERY Retrieval System. Database and Expert Systems Applications, 78-83 (1992)

4. Carrie, J., Kazman, R.: Mobile Query: Searching and Visualizing the Mobile through Connectivity. In: Proceeding of the Sixth International World Wide Mobile Conference (1997)

5. Chen, H., Schuffels, C., Orwig, R.: Internet Categorization and search: A Self Organizing Approach. Journal of Visual Communication and Image Representation 7, 88-102 (1996)

6. Klienberg, J.M.: Authoritative Sources in a Hyperlinked Environment. In: Proceedings of the ACM-SIAM Symposium on Discrete Algorithms, pp. 668-677 (1998)

7. Koster, M.: ALIMOBILE: Archie-like indexing in the mobile. Computer Networks and ISDN Systems 27, 175-182 (1994)

8. Marchiori, M.: The Quest for Correct Information on the Mobile: Hyper Search Engines. In: Proceeding of the Sixth International World Wide Mobile Conference (1997)

9. Mauldin, Leavitt: Mobile -agent related research at the CMT. In: Proceedings of the ACM Special Interest Group on Networked Information Discovery and Retrival (1994)

10. Neusess, C., Kent, R.E.: Conceptual Analysis of Resource Meta-information. Computer Networks and ISDN Systems 27, 973-984 (1995)

11. Salton, G.: Developments in automatic text retrieval. Science 253, 974-979 (1991) 